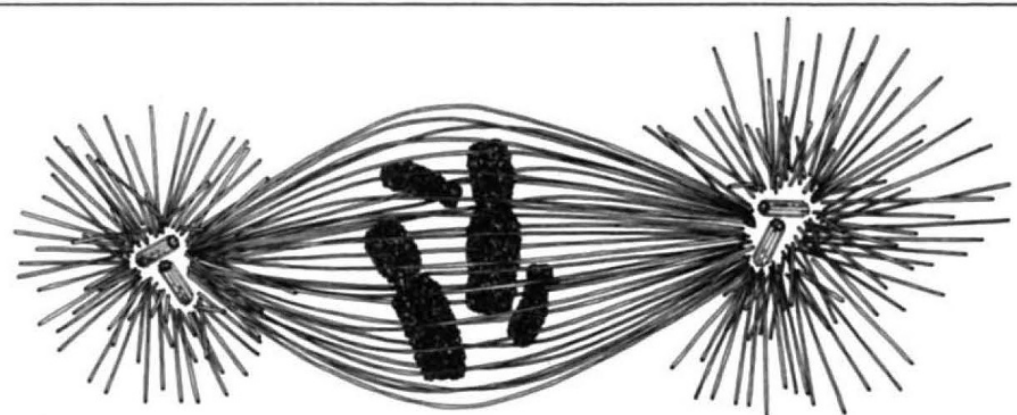

The mitotic apparatus of a cell in metaphase. Chromosomes, each divided into a pair of chromatids joined only at the centromere, sit in the centre of a spindle composed of microtubules. Some microtubules connect the kinetochores on the centromeres to a centrosome; others connect the two centrosomes, each composed of a pair of centrioles surrounded by amorphous pericentriolar material. From Cell and Molecular Biology by E.J. Dupraw (Academic, 1968).

the spindle.

The autoantibodies of most value in the study of kinetochores come from the sera of a subset of rheumatoid patients, those with the so-called CREST (calcinosis, Raynaud's phenomenon, oesophageal dysmotility, sclerodactylyl, telangiectasia) variant of scleroderma. Such sera, when used as immunofluorescent probes on frozen tissue sections or cultured cell lines, give a characteristic speckled nuclear fluorescence. In mitotic cells, fluorescence is clearly seen to be localized to the centromere region of each chromosome. Immunoelectron microscopy has localized the binding of one CREST serum to the inner and outer plates of the trilaminar kinetochore but not to the underlying centromeric chromatin ${ }^{2}$ and has been used to identify 'prekinetochores' during interphase, the period when they are normally cytologically invisible. Like the centrosome, these small electron-dense spheres duplicate and separate during the $G_{2}$ phase of the cell cycle.

Two recent papers have used CREST sera to probe both the molecular composition of the kinetochore and its integration into the chromosome. In one, twelve antisera that stained kinetochores were shown, by immunoblotting, to recognize a 77,000-molecular-weight protein; nine of the sera also recognized an antigen of molecular weight 110,000 (ref. 3). Interestingly, both proteins were present not only in isolated chromosomes but also in chromosome 'scaffolds', the protein framework which persists when metaphase chromosomes are first depleted of histones and then digested with nucleases. In the second paper, other CREST sera were shown to recognize a different spectrum of presumptive kinetochore components ${ }^{4}$. At least four proteins, of $14,000,20,000$, 23,000 and 34,000 molecular weight, are detectable in both interphase and mitotic cells of various cultured lines, suggesting that the structural re-organization of the kinetochore at prophase, which parallels its competence to interact with microtubules, is not accompanied by major compositional changes ${ }^{4}$. The exciting possibility that one or more of these proteins undergoes some sort of cell-cycledependent modification is now experimentally testable.

The anti-kinetochore specificity of CREST sera has also been useful in investigating the role of the kinetochore as a microtubule-organizing centre. Preincubation of lysed mitotic cells with antiserum before incubation with tubulin specifically blocked the assembly of microtubules onto the kinetochore whilst having no effect on growth onto the centrosome ${ }^{4}$. Although there are various interpretations of this finding, the most intriguing is that the two presumptive centres are chemically, and possibly functionally, distinct. An assessment of the extent and significance of the distinction should be helped by those scleroderma patients (a low percentage) who produce autoantibodies not to kinetochores but to centrosomes 5 . One such

\section{Astrophysics}

\section{from A.G. Lyne}

THE origin of the intense magnetic fields of many neutron stars is not clearly understood. Until recently they have commonly been thought to originate from the field of a few gauss of the presupernova stars from which neutron stars evolve; conservation of magnetic flux during the violent collapse of a presupernova star to a neutron star would result in the $10^{12} \mathrm{G}$ surface field strength of the latter that is needed to provide a satisfactory explanation of the spin down, radio emission and polarization of radio pulsars, if they are neutron stars. Now Blandford, Applegate and Hernquist have suggested a quite different origin of the magnetic field of neutron stars by demonstrating, theoretically, two thermally driven instabilities in which a reasonably modest seed field in the neutron star may be amplified to $10^{12} \mathrm{G}$ by the flow of heat from the interior of the star as it cools following supernova collapse ${ }^{1}$. Their proposal has a serum has now been characterized cytologically ${ }^{6}$. By light and electron immunocytochemistry it stains the cloud of unstructured fuzz that surrounds the two centrioles - the so-called pericentriolar material, which appears to be more involved than the centriole in initiating microtubule assembly. The pericentriolar material remains closely associated with the centrioles, staining as a single bright dot which splits as the cell enters division. In the early divisions of mouse embryo cells, from which centrioles are absent, the pericentriolar material has a different fate, occupying a broad band at the spindle pole during mitosis but becoming undetectable during interphase.

The cellular control of microtubule assembly clearly resides in a limited number of preformed sites, the activity of which is tightly integrated with the cell cycle. The scleroderma sera described here offer the first real molecular probes of these putative microtubule-organizing centres and we shall doubtless be hearing a lot more about them in the next two or three years.

1. Rieder, C. L. Int. Rev. Cytol, 79, 1 (1982).

2. Brenner, S., Pepper, D., Berns, M. W. Tane E. \& Brinkley, B. R. J. Cell Biol. 91, 95 (1981).

3. Earnshaw, W. C., Halligan, N., Cooke, C. \& Rothfield, N. J. Cell Biol. 98,352 (1984)

4. Cox, J. V., Schenk, E. A. \& Olmsted, J. B. Cell 35, 331 (1983).

5. Tuffanelli, D. L., McKeon, F., Kleinsmith, D. K., Burham, T. K. \& Kirschner, M. Arch. Derm. 119, 560 (1983).

6. Calarco-Gillam, P. D., Siebert, M. C., Hubble, R., Mitchison, T. \& Kirschner, M. Cell 35, 621 (1983).

Jeremy S. Hyams is at the Department of Botany and Microbiology, University College London, Gower Street, London WCIE 6BT.

\title{
Origins of the magnetic fields of neutron stars
}

number of attractions and a number of problems.

Neutron stars are believed to consist of a superfluid superconducting core inside a solid crust, a few kilometres thick, which is not superconducting. Any field present in the core at the time of formation will be trapped there, essentially forever. However, within a kilometre or so of the surface, the electrical conductivity is low enough that ohmic losses could dissipate any embedded magnetic field within a few million years and there is increasing evidence that the magnetization of neutron stars does, indeed, decay ${ }^{2}$.

Assuming that such decay is occurring, Blandford et al. argue that the magnetic field of neutron stars must be confined to the surface layers where it can decay. This is just the region where the two instabilities described by Blandford et al. will enhance the magnetic field. The evolution of an iso- 